\section{The Biological Perspective} Ideas from New Green Chemistry Concepts for Improving the Performance of Microalgae

\section{by Christian Wilhelm, University of Leipzig}

The generation of biofuel by producing, harvesting and processing microalgal biomass has not yet reached a positive energy balance as shown by most of the published life cycle studies. The major reasons are the low energy efficiency of the photon-to-biomass conversion and the high energy demand for mixing during cultivation, harvesting, extraction and refining. The energy efficiency of microalgal biomass production is not limited by photosynthesis, but by the metabolic processes for allocating carbon into the macromolecular pools of the algae cell. The proposed concept of "new green chemistry" tries to reduce the number of conversions to a minimum in order to increase energy efficiency.

\section{Introduction}

Microalgal mass cultures have attracted new attention during the last decade as a source of new feedstock, of biofuels (biodiesel, methane or bioethanol) and of (highly) valuable products (e.g. carotenoids, polyunsaturated fatty acids or other food additives) having the potential to help build a future green economy (Olaizola 2003). The technical and economic feasibility of the microalgaebased pathway to produce high-value products depends mainly on the costs of biomass production and refinement and the price of the product (Stephens et al. 2010). Microalgal technology will be economically feasible if technological progress reduces the costs of the entire process from photon to the final product to below the amount that is derived from fossil carbon. However, economic feasibility is not climate friendly per se. In the case of biofuels, not only the price but also a life cycle analysis (LCA) must show that the ratio of usable megajoules of energy per unit of greenhouse gas emission is lower than for fossil sources. The first generation of biofuels (bioethanol from sugarcane or biodiesel from rape seeds) shows a reduction in greenhouse gas emissions by only about $30 \%$ compared to fossil oil (Kaltschmitt, Hartmann 2009). On the one hand, algae can offer important advantages over first generation biofuels, such as much higher productivity per area, no demand for arable land, higher water use efficiency, and improved nutrient recycling by employing integrated waste water concepts. On the other hand, closed photobioreactors used for growing algae consume large amounts of electric energy for mixing, harvesting and downstream processes. Since the electric energy itself is not produced free of $\mathrm{CO}_{2}$, the whole process from photon capture to biofuel creation emits more $\mathrm{CO}_{2}$ than is fixed by the algae. At the present state of the art, algae-derived biofuels emit more greenhouse gases than do fuels produced from fossil sources (Weinberg et al. 2012). Although the feasibility of microalgal technology depends on the optimization of all technological steps, it is the task of this study to analyse the first step, namely the physiological limits of the photon-to-biomass conversion and to identify the bottlenecks in this process and the opportunities for increasing the physiological energy efficiency of microalgae. The future challenge is to optimize the whole process in a way that the greenhouse gas emissions are reduced far below the level of fossil oil and to increase the productivity per unit area by at least one order of magnitude. At present, the major route in this direction is the one called "green chemistry".

\section{Classical Green Chemistry}

According to Anastas and Warner (1998), green chemistry is "the utilisation of a set of principles that reduces or eliminates the use or generation of hazardous substances in the design, manufacture and application of chemical products" (Anastas, Warner 1998). One of the principles is to use raw material or feedstock from renewable sources. Since photosynthesis is the only process which delivers new organic carbon from renewable sources with present-day technologies, the process can be divided into three major steps: (a) biomass formation, (b) harvest and transport, and (c) the conversion of biomass into the product. In the first process, the activity of the photosynthetic machinery in the green plants generates organic 
carbon from inorganic $\mathrm{CO}_{2}$, water and nutrients. These primary photosynthetic products, mainly sugars, are then converted by metabolic processes into complex biological macromolecules such as lipids, proteins and high molecular carbohydrates (e.g. starch, cellulose and hemicelluloses). In higher plants, lignin is an additional macromolecule that is not only important in trees but also in all annual plants, because the stability of the shoot and the water transport capacity inside the plant depend on lignin-stabilized cells. The lipids and the proteins are biologically active components because they deliver the membranes and enzymes for the metabolic routes and the reaction matrix for cell growth. The metabolic conversion of sugars into lipids and proteins requires additional nutrients, e.g. nitrogen, for the production of amino acids, the monomers of proteins. In higher plants, new biomass is mainly produced as shoots, roots and leaves, but energy storage is mainly in seeds as lipid or as starch, so reallocation of carbon is required. The formation of biomass includes many biochemical reactions and inevitably depends on the nutrient supply (e.g. nitrogen and phosphate) which are not part of the final product. During the biomass-to-product conversion these elements have to be eliminated and recycled. In the case of microalgae, the situation is more advantageous because the total amount of carbon can be harvested and the biomass can be completely converted into the product, whereas in higher plants significant amounts of the biomass remain as remnants (e.g. non-degradable lignin).

The main investments in creating algal biomass are due to the necessity of mixing, which is important for preventing $\mathrm{CO}_{2}$ limitation and $\mathrm{O}_{2}$ oversaturation (a high oxygen concentration inhibits the carboxylation process) and for providing the cells with the appropriate amount of light energy. This is very crucial for the overall photosynthetic efficiency. Further energy is needed for nitrogen fertilization. The nitrogen demand of fast growing algae is $1 \mathrm{~N}$ per $7 \mathrm{C}$. Based on the facts that the energy demand for the assimilation of 7 mole $\mathrm{C}$ is $805 \mathrm{kcal}$ and the energy demand for the assimilation of 1 mole $\mathrm{N}$ is $289 \mathrm{kcal}$, it is obvious that about $35 \%$ of the energy has to be invested in nitrogen metabolism.
Microalgae can be harvested either by centrifugation, filtration or flocculation. The major challenge posed by this process results from the fact that the biomass concentration is very low compared to the amount of water to be treated. In the best case, an algae suspension contains 20-40 g biomass per litre, equal to 96-98\% water. This "low" cell density is needed because the cells absorb the light very efficiently and a more highly concentrated suspension will lead to extreme self-shading in the bioreactor. Harvesting is therefore associated with high mass transfer against high back pressure in the case of filtration. Centrifugation is extremely energy consuming, and flocculation makes further re-treatment of nutrient solution necessary before the solution can be re-used for algal growth. Even if one admits that intelligent technologies can improve the efficiency, harvesting has to solve a basic physical problem: the concentration of energy. Light from the sun reaches the surface in a much "diluted" form (e.g. $1000 \mathrm{~W} / \mathrm{m}^{2}$ year photosynthetically active radiation). A car with a surface area of about $8 \mathrm{~m}^{2}$ consumes about 15001 diesel per year. Even if the efficiency from photon to tank would be $5 \%$, the area needed to provide the car with the requested energy is about 30 ha. This simple calculation illustrates the tremendous demand on area, which leads to the basic problem of mass transfer over long distances.

The third process is the conversion of biomass to biofuels. Starch is degraded by enzymatic activity to glucose and then fermented to ethanol. The result is a mixture of water and ethanol. Most of the energy used to produce bioethanol is needed in the final distillation step to obtain highly concentrated (nearly water-free) ethanol. In the case of biodiesel, the oil from algae or oil seeds is extracted with the help of a lipophilic solvent (e.g. butanol). Since plant oils contain one glycerine molecule per three fatty acids, the lipid must be transesterified with methanol or ethanol. Here again, the transesterification process consumes the largest amount of energy in this final step. In the case of algal oils, the percentage of sulpholipids or glycolipids can be increased, delivering only two fatty acids per glycerine. 


\section{Challenges and Limits to Improving the Efficiency of Biomass Formation}

Many authors have emphasized that microalgae are more efficient than higher plants for producing biomass. In contrast to higher plants, algae can be grown independently of arable land, its water use efficiency (demand for water per $\mathrm{kg}$ biomass produced) is at least twenty times as good and the yields per area and year are higher by several factors. Some authors claim that the productivity of algae per unit area is 10-100 times higher than that of higher plants (see Schenk et al. 2008; Brennan, Owende 2010 and references therein). An improvement on the present-day state of the art can be expected because only a few species have been used so far although algal biodiversity is very high and has not yet been exploited biotechnologically. A second reason that has been put forward is that the photosynthetic efficiency can be improved not only by a better reactor design but also by metabolic engineering (Schuhmann et al. 2012). Table 1 summarizes the potential of microalgae and agricultural plants.

Table 1: Yield per area and year of plant production systems for biofuels

\begin{tabular}{lcc}
\hline & $\begin{array}{c}\text { Area demand } \\
{\left[\mathrm{m}^{2} \text { year }\right.} \\
\mathrm{kg} \text { biodiesel] }\end{array}$ & $\begin{array}{c}\text { Biofuel productivity } \\
{[\mathrm{kg} \text { biodiesel] }} \\
\text { ha year] }\end{array}$ \\
\hline Maize & 66 & 152 \\
Soybean & 18 & 562 \\
Rape seed & 12 & 862 \\
Palm oil & 2 & 4.7 \\
Microalgae & 0.1 & 86.5 \\
\hline
\end{tabular}

Source: Based on data of Mata et al. 2010

In the last decade, we have developed the experimental know-how to achieve an energy balance in the biological conversion from photon to biomass. Since all the cells in a microalgal culture are photosynthetically active, it is possible to follow the fate of the absorbed energy through the major metabolic pathways in detail. This is not possible for higher plants because they consist of tissues with different physiological functions with very complex sink and source carbon allocation, which does not permit us to determine complete energy balances.

\section{From Photon to Biomass and Its Conver- sion Efficiency}

Energy balancing is based on a bio-optical model which measures the energy input and energy storage at four different levels and compares the energy savings monitored by the bio-optical model with the biomass that has actually been formed. The balance is based on optimal growth conditions under steady state conditions, and the comparison of modelled and measured biomass formation is based on daily production given a 14-h illumination period and $10 \mathrm{~h}$ of darkness. The input parameter is the number of absorbed quanta per cell or chlorophyll molecule per day $\left(\mathrm{Q}_{\text {phar }}\right)$. $\mathrm{Q}_{\text {phar }}$ is different from the incident photosynthetic active radiation $\left(\mathrm{Q}_{\mathrm{par}}\right)$ because the number of photons that reach a cell depends on the cell density, the light gradient inside the reactor, the mixing velocity, the light spectrum, and the absorption spectrum of the cell. For a good energy balance, the experimental conditions must be chosen in such a way that the amount of $\mathrm{Q}_{\text {phar }}$ does not depend on unverified assumptions. Therefore, we have used a flat cuvette with a very low cell density leading to a minimal light gradient inside the cuvette. This makes it possible to measure $\mathrm{Q}_{\text {phar }}$ in quantitative terms if the path length in the cuvette, the light spectrum, the absorption spectrum, the chlorophyll content, and the number of cells are known. Then $\mathrm{Q}_{\text {phar }}$ can be calculated using equation 1 :

$$
Q_{\text {phar }}=\int_{400 m}^{700 m} Q(\lambda)-Q(\lambda) e^{\left.-a^{*} p h y(\lambda) \times[\mathrm{Chl} l] \times d\right)} d \lambda
$$

[Chl a] is the chlorophyll concentration in the cuvette $\mathrm{d}=$ the path length in the cuvette

$\mathrm{a}^{*}{ }_{\text {phy }}=$ the chlorophyll-specific absorption coefficient "a* ${ }_{\text {phy }}$ " is defined by equation 2 :

$$
a_{p h y}^{*}(\lambda)=\frac{2.3 \times A(\lambda)}{d \times[\text { Chla }]}
$$

The coefficient " $a *$ " " defines the absorptivity of the cells. It can be experimentally obtained by dividing a scatter-corrected absorption spectrum by the chlorophyll concentration. The formal dimension of $\mathrm{a}^{*}$ phy is $\mathrm{m}^{2} / \mathrm{mg}$ chlorophyll and gives the area available for each chlorophyll molecule. 
The more densely packed the chlorophyll inside the cell, the lower the chance that the chlorophyll can hide from a photon. Therefore, the amount of chlorophyll needed to absorb a given amount of photons at a given light flux strongly depends on $\mathrm{a}^{*}{ }_{\text {phy }}$, which declines as a function of cell size as well as of the chlorophyll content per cell (Blache et al. 2011). Since the latter is strongly dependent the light acclimation status, the absorptivity of the cell suspension changes in the natural light climate. Toepel et al. (2005) have shown that $\mathrm{a}^{*}{ }_{\text {phy }}$ can vary under natural conditions by a factor of two.

The next energetic conversion step is the photochemical reaction. Its efficiency can be measured as photosynthetic quantum yield expressed as the number of transported electrons per number of absorbed photons. The quantum yield of photosystem II is optimally about $80 \%$, which is slightly lower than the $90 \%$ achieved in photosystem I. With increasing light intensity, the overall quantum yield decreases, because the photochemical reaction kinetics are about three orders of magnitude faster than the biochemical processes. As a consequence, at a higher light intensity the acceptor side of the photosystems is in a reduced state and the quantum yield for fluorescence and heat emission rises. Since chlorophyll molecules in the excited state can transfer the energy to molecular oxygen forming reactive oxygen species (ROS) such as singlet oxygen or superoxide radical anions, which are extremely toxic, evolution has developed photoprotection mechanisms against photoinhibition. In short, the major mechanism is to convert the absorbed photons into heat by a so-called non-photochemical quenching (NPQ) mechanism. NPQ can be quantified by the socalled pulse-amplitude-modulated fluorescence analysis (Büchel, Wilhelm 1993). This method, developed by Schreiber et al. (1986), allows the photosynthetic quantum efficiency to be measured in situ. Multiplying the quantum yield times the absorbed quanta gives the number of electrons per chlorophyll molecule per time, which can be measured independently by oxygen evolution. Therefore, photosynthetic electron flow can be measured by two independent methods, making it possible to assess the electron use efficiency.

The fluorescence-based electron transport rates reflect the bulk of electrons extracted from water, whereas the oxygen production represents the gross balance from oxygen-producing and oxygen-consuming processes. The latter does not contain only the ongoing mitochondrial respiration but also all light-driven oxygen-consuming processes which are summarized as "alternative electron transport". These include photorespiration and the so-called Mehler reaction. In this case, electrons delivered from photosystem I are transferred to molecular oxygen thereby producing $\mathrm{O}_{2}^{-}$instead of reducing $\mathrm{NADP}^{+}$to $\mathrm{NADPH}+\mathrm{H}^{+}$. Thus, oxygen is consumed and the oxygen superoxide anion is detoxified via hydrogen peroxide to water. This water-water cycle (Asada 1999) is not completely futile because it generates ATP. Under real photobioreactor conditions, photorespiration can be suppressed by providing a sufficient $\mathrm{CO}_{2}$ supply, whereas the water-water cycle remains operative. Comparison of fluorescence-based electron transport rates and oxygen production makes it possible to quantify the alternative electron transport. Figure 1 shows that, in a dynamic light climate mimicking a sunny day, fluorescence and oxygen evolution match perfectly in the early morning and in the late afternoon, when the light intensity is relatively low. In full sunlight, in contrast, about one third of the energy is lost by alternative electron cycling.

Finally, the carbon assimilation efficiency has to be quantified. It determines the fraction of electrons which are finally stored in the biomass. Here again, comparison of the different independent methods can improve our understanding of the underlying physiological mechanisms. The assimilation efficiency is influenced by three major processes:

- carbon losses by respiration and biochemical pathways that include decarboxylation reactions (see fig. 2);

- electron transfer to other elements than carbon or by reducing carbohydrates to more reduced components, e.g. lipids;

- carbon losses due to carbon excretion.

The carbon loss can be measured by respirometry. Here, the $\mathrm{O}_{2} / \mathrm{CO}_{2}$ ratio can be continuously measured in the light and in the dark. For instance, this ratio rises if the cell stores the assimilated carbon in lipids instead of in starch. The carbon allocation can also be measured by a new procedure based on 
Fig. 1: The electron transport rates during a dynamic daily light climate



Note: Dynamic daily light climate (light intensity dashed line) measured by oxygen evolution (solid black line) and by fluorometry (dotted grey line). The difference between fluorescence- and oxygen-based electron transport rates reflects the activity of alternative electron cycling, indicating the loss of reductants which cannot be used for carbon assimilation, but which might contribute to ATP biosynthesis (Mehler reaction).

Source: Own compilation

quantitative chemometry of FTIR spectra (Wagner et al. 2010). This approach enables us to quantify the rate of biosynthesis of proteins, carbohydrates and lipids in parallel with a time resolution of minutes. These measurements can be quantitatively related to the amount of new biomass based on the growth rate.

In figure 2, the metabolic pathway is shown from the primary photosynthates in the Calvin cycle to the major macromolecular pools in typical green algae, e.g. Chlorella. In some algae, the cell wall does not contain cellulose, so the carbon needed for the formation of cell walls is reduced or is replaced by other macromolecules, e.g. proteins apparatus to cellular macromolecules

Source: Own compilation in the case of Chlamydomonas. From these pathways it is obvious that the synthesis of lipids and proteins includes decarboxylation steps, which not only lead to the loss of carbon but also to an increase in the metabolic costs for new cell formation. These metabolic costs have to be taken into account in a full energy balance comprising photon to biomass.

We have performed such complete energy balances for different species of green algae and diatoms under different experimental conditions (Wagner et al. 2005; Jakob et al. 2007).

Fig. 2: Carbon/electron flow from the photosynthetic

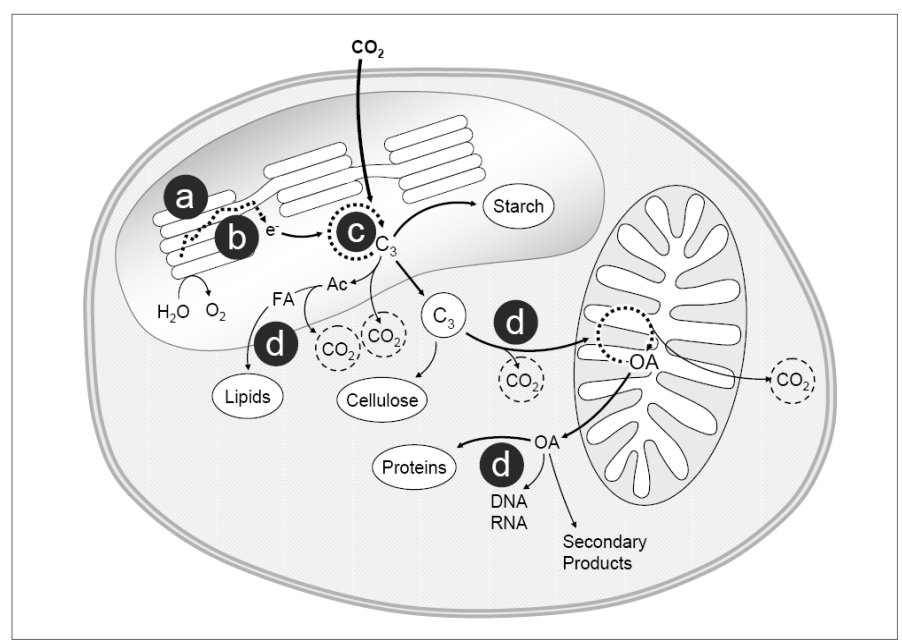

Note: Electrons are generated by a water-splitting apparatus in the thylakoid membrane (a). Electron flow from water to NADPH generates ATP (b) needed for $\mathrm{CO}_{2}$ fixation in the Calvin cycle (c) producing $\mathrm{C}_{3}$ sugar. These sugars can be stored in the chloroplast as starch or converted to fatty acids (FA) which are exported to the cytosol and stored in lipid vesicles. Exported $\mathrm{C}_{3}$ sugars can be metabolized in the cytosol to feed the citric acid cycle in the mitochondrion, which delivers the carbon skeletons for the synthesis of amino acids (proteins), nucleic acids and secondary products (d). The transformation of $\mathrm{C}_{3}$ sugars to $\mathrm{FA}$ as well as to amino acids is always linked with decarboxylation reactions increasing the electron demand per carbon fixed in the biomass. 
The highest photon-to-carbon conversion efficiency is found under light acclimated conditions at a light level where the cells absorb a number of photons per chlorophyll that is close to the inclination point of the light saturation curve. At this light intensity, the growth rate is only half of the maximum. However, even under these conditions the photon to biomass conversion efficiency, expressed as $\mathrm{P} / \mathrm{C}$, is about $30 \%$ above the theoretical optimum, but only if the macromolecular composition of the cells is not favourable for biofuel production (see Fig. 3). Increasing the yield of oil or protein production is inevitably linked to much lower photon-to-biomass conversion efficiencies, which decrease further when the light field cannot be fully controlled, leading to light acclimation reactions in the cell. The data show that the potential for improving the photon-to-biomass conversion is not restricted by the photosynthetic efficiency but limited by the metabolic capacity of the cells to convert sugar into the biochemically complex matrix of a growing cell. There is one exception, namely the decrease in algal absorptivity as a result of the minimization of the light harvesting antennae can reduce the losses from NPQ. However, this will not lead to higher productivity but to a much lower energy input for mixing or to savings in reactor cost by making less light dilution necessary.

The concept of new green chemistry was developed on the basis of these results.

\section{New Green Chemistry}

New green chemistry tries to reduce the number of conversion steps from $\mathrm{CO}_{2}$ to the final product. This strategy is shown in figure 4 using the production of isoprene as an example. The production pathway for traditional green chemistry is shown on the left hand side, whereas the concept of new green chemistry is depicted on the right.

The basic idea is to use the algal cell as a cell factory that uses its biosynthetic machinery to produce metabolites. Therefore, the primary photosynthetic products (sugar or organic acids) are not directed to produce biomass, but to be excreted. The excretion product can be either a bulk chemical, which has to be processed further outside the cell, or it can be a product that is formed in the cell in a metabolically designed pathway. In this design, the technical and energetic input for harvesting is strongly reduced. Since the formation of

Source: Based on the data of Langner et al. 2009 
new biomass is suppressed, the cells do not need large amounts of nutrients, which are necessary for the build up of cellular macromolecules. If the maximization of biomass productivity is no longer the main target for bioreactor optimization, the cells can be managed in a completely different environment. The cells can be attached to a surface, forming an active biofilm which is thick enough to absorb $90 \%$ of the light, which must be kept wet, and which must permit carbon dioxide to enter for photosynthetic assimilation. If the excretion product is water soluble, only a low volume of water flow is necessary to control the optimal environment for the cells and to withdraw the metabolite from the cells to prevent inhibitory reactions. In this set-up, a complete refinery concept can be integrated if the cellular metabolic pathway can be used to produce the final product within the cell. The best option as a potential product is a substance which is easy to gasify, so that the "concentration process" can be done by minimal mass transfer.

In the example of isopentenyl diphosphate (IPP), which is needed for many compounds in the cell (e.g. carotenoids), seven enzymatic steps are needed to convert the primary products from photosynthesis into IPP. This pathway can consume up to $30 \%$ of the assimilated carbon to pro- duce xanthophylls. The addition of one enzyme, the isoprene synthase, is sufficient to funnel part of this metabolite flow into isoprene. At physiological temperatures, isoprene is a gas that is emitted from the cells without any barrier. If the cells are exposed to a constant flow of air, which is needed to feed the cells with $\mathrm{CO}_{2}$ and to dilute the rising oxygen concentration due to photosynthetic activity, isoprene will leave the photobioreactor by the normal gas stream. If this gas stream enters cold water, i.e. water below the condensation temperature for isoprene (about $5-10^{\circ} \mathrm{C}$ ), it becomes liquid and can be easily separated from water by phase partitioning. The activity of isoprene synthase has to be regulated in such a way that the total carbon loss from respiration at night and from the emission of isoprene when it is light is more or less equivalent to the carbon gained by photosynthetic carboxylation.

\section{Conclusions}

Complete energy balances from photon to harvestable biomass can help to identify the limiting processes of carbon fixation at the cellular level of microalgae. The results show that the activity of enzymes has to be regulated to obtain high productivity and to reduce total carbon loss from respiration at night. The overall efficiency of biomass formation can be improved by innovative photobioreactor design that is kinetically adapted to rate-limiting steps in cell physiology. However, taking into account the real photon demand per assimilated carbon and the energy input for biorefinement, it becomes obvious that instead of improving growth, the cell metabolism has to be regulated to establish a new homoeostasis be-

Source: Own compilation 
tween carboxylation and biosynthesis. Cells must, therefore, be selected which possess high physiological activity in the attached state in a biofilm and which can actively drive photosynthesis with a minimal demand for protein and phosphate for cell maintenance. This research is at its very beginning, and it is not very likely that commercial applications will be ready for investment in the short term. However, recent progress in the approach of new green chemistry shows that this approach is feasible and that the potential for investing more intellectual and scientific input into this strategy seems to be very promising.

\section{References}

Anastas, P.; Warner, J.C., 1998: Green Chemistry: Theory and Practice. New York

Asada, K., 1999: The Water-water Cycle in Chloroplasts: Scavenging of Active Oxygens and Dissipation of Excess Photons. In: Annual Review of Plant Physiology and Plant Molecular Biology 50 (1999), pp. 601-639

Blache, U.; Jakob, T.; Su, W. et al., 2011: The Impact of Cell-specific Absorption Properties on the Correlation of Electron Transport Rates Measured by Chlorophyll Fluorescence and Photosynthetic Oxygen Production in Planktonic Algae. In: Plant Physiology and Biochemistry 49/8 (2011), pp. 801-808

Brennan, L.; Owende, P., 2010: Biofuels from Microalgae - A Review of Technologies for Production, Processing, and Extractions of Biofuels and Co-products. In: Renewable Sustainable Energy Reviews 14/2 (2010), pp. 557-577

Büchel, C.; Wilhelm, C., 1993: In-vivo Analysis of Slow Fluorescence Induction Kinetics in Algae: Progress, Problems and Perspectives. In: Journal Photochemistry and Photobiology 58/1 (1993), pp. 137-148

Jakob, T.; Wagner, H.; Stehfest, K. et al., 2007: A complete energy balance from photons to new biomass reveals a light- and nutrient-dependent variability in the metabolic costs of carbon assimilation. In: Journal of Experimental Botany 58 (2007), pp. 2101-2113

Kaltschmitt M.; Hartmann, H. (eds.), 2009: Energie aus Biomasse. Berlin

Langner, U.; Jakob, T.; Stehfest, K. et al., 2009: An energy balance from absorbed photons to new biomass for Chlamydomonas reinhardtii and Chlamydomonas acidophila under neutral and extremely acidic growth conditions. In: Plant Cell and Environment 32 (2009), pp. 250-258
Mata, T.M.; Martins, A.A.; Caetano N.S., 2010: Microalgae for Biodiesel Production and Other Applications. A Review. In: Renewable and Sustainable Energy Reviews 14 (2010), pp. 217-232

Olaizola, M., 2003: Commercial Development of Microalgal Biotechnology: From the Test Tube to the Market Place. In: Biomolecular Engineering 20 (2003), pp. 459-466

Schenk, P.M.; Thomas-Hall, S.R.; Stephens, E. et al., 2008: Second Generation Biofuels: High Efficiency Microalgae for Biodiesel Production. In: BioEnergy Research 1 (2008), pp. 20-43

Stephens, E.; Ross, I.L.; King, Z. et al., 2010: An Economic and Technical Evaluation of Microalgal Biofuels. In: Nature Biotechnology, 28 (2010), pp. 126-128

Schuhmann, H.; Lim, D.K.Y.; Schenk, P.M., 2012: Perspectives on Metabolic Engineering for Increased Lipid Contents in Microalgae. In: Biofuels 3 (2012), pp. 71-86

Schreiber, U.; Schliwa U.; Bilger W., 1986: Continuous Recording of Photochemical and Non-photochemical Chlorophyll Fluorescence Quenching with a New Type of Modulation Fluorometer. In: Photosynthesis Research 10 (1986), pp. 51-62

Toepel, J.; Langner, U.; Wilhelm, C., 2005: The Combination of Flow Cytometry and Single Cell Absorption Spectroscopy to Study the Phytoplankton Structure and to Calculate the $\mathrm{Chl}$ a Specific Absorption Coefficients at the Taxon Level. In: Journal of Phycology 41 (2005), pp. 1099-1109

Wagner, H.; Jakob, T.; Wilhelm, C., 2005: Balancing the Energy Flow from Captured Light to Biomass under Fluctuating Light Conditions. In: New Phytologist 169 (2005), pp. 95-108

Wagner, H.; Stehfest, K.; Jakob, T. et al., 2010: The Use of FTIR Spectroscopy to Assess Quantitative Changes in the Biochemical Composition of Microalgae. In: Journal of Biophotonics 3 (2010), pp. 557-566 Weinberg, J.; Kaltschmitt, M.; Wilhelm C., 2012: Biofuels from Microalgae - An Environmental Analysis. In: Biomass Conversion and Biorefinery (submitted)

\section{Contact}

Prof. Dr. Christian Wilhelm

Institute of Biology

University of Leipzig

Johannisallee 23, 04103 Leipzig, Germany

E-mail: cwilhelm@rz.uni-leipzig.de

Internet: http://www.uni-leipzig.de/ pflaphys/ 